\title{
OPTIMIZATION OF THE TEST INTERVALS OF A NUCLEAR SAFETY SYSTEM BY GENETIC ALGORITHMS, SOLUTION CLUSTERING AND FUZZY PREFERENCE ASSIGNMENT
}

\author{
E. ZIO",1,2 and R. BAZZO ${ }^{2}$ \\ ${ }^{1}$ Ecole Centrale Paris- Supelec, Paris, France \\ Chair "Systems Science and Energetic Challenge" \\ European Foundation for New Energy - EDF \\ ${ }^{2}$ Politecnico di Milano, Milano, Italy, Dipartimento di Energia \\ "Corresponding author. E-mail : enrico.zio@ecp.fr, enrico.zio@supelec.fr
}

Received December 11, 2009

Accepted for Publication May 06, 2010

In this paper, a procedure is developed for identifying a number of representative solutions manageable for decision-making in a multiobjective optimization problem concerning the test intervals of the components of a safety system of a nuclear power plant. Pareto Front solutions are identified by a genetic algorithm and then clustered by subtractive clustering into "families". On the basis of the decision maker's preferences, each family is then synthetically represented by a "head of the family" solution. This is done by introducing a scoring system that ranks the solutions with respect to the different objectives: a fuzzy preference assignment is employed to this purpose. Level Diagrams are then used to represent, analyze and interpret the Pareto Fronts reduced to the head-of-the-family solutions.

KEYWORDS : Multiobjective Optimization, Pareto Front, Pareto Set, Subtractive Clustering, Level Diagrams, Fuzzy Preference Assignment, Fuzzy Preference Scoring, Genetic Algorithms, Test Intervals Optimization, Safety System, Nuclear Power Plant

This work has been partially funded by the Foundation pour une Culture de Securité Industrielle of Toulouse, France.

\section{INTRODUCTION}

The task of selecting preferred solutions by a decision maker (DM) confronted with multiple objectives can be difficult when dealing with large Pareto Fronts and Sets, representative of the best (non-dominated) solutions of the problem at hand. To make the task feasible, only a small number of solutions representative of the Pareto Front and Set should be offered for selection to the DM.

Aid to multiobjective decision making can come from methods that provide more flexible ways of handling multiple objectives and DM preferences for optimal decision making than the quasi-prescriptive methods based on the aggregation of the multiple objectives into a single one. In this respect, the importance for the practice of multiobjective decision making and optimization of determining and including DM preferences is inarguable.

The particular context of reference for this work is practical decision making situations concerning the highconsequence technologies involved in the nuclear power field. The starting point is the acknowledgment that technical analyses provide useful decision support in the sense that their outcomes inform the decision makers insofar as the technical side of the problem is relevant for the decision.

It is further understood that the actual decision outcome for a critical situation involving a potential for large consequences typically derives from a thorough process which combines i) an analytic evaluation of the situation (i.e., the technical assessment) by rigorous, replicable methods evaluated under protocols agreed upon by an expert community and peer-reviewed to verify the assumptions underpinning the analysis, and ii) a deliberative group exercise in which all involved stakeholders and decision makers collectively consider the decision issues, look into the arguments for their support, scrutinize the outcomes of the technical analysis and introduce all other values (e.g. social and political) not explicitly included in the technical analysis. This way of proceeding allows us to keep the technical analysis manageable by complementation with deliberation for ensuring coverage of the non-modelled issues. In this way, the analytic evaluation (i.e., the technical assessment) supports the deliberation by providing numerical outputs of the relevant parameters, possibly to be compared with predefined numerical safety criteria for further guidance to the decision, and also all the arguments 
behind the analysis itself, including the assumptions, hypotheses, parameters and their uncertainties. The ultimate concern of the DM is to fulfil his or her conflicting objectives, while satisfying the constraints posed by the problem itself.

In practice, the technical assessment leads to a multiobjective optimization problem whose solution is provided in terms of a discrete approximation of the Pareto Front and the identification of the corresponding Pareto Set of solutions. The ultimate purpose of the technical assessment is to provide the decision makers with a clearly informed picture of the problem upon which they can confidently reason and deliberate. On the basis of the information provided by the technical assessment, the decision maker (DM) is requested to select one or more feasible solutions according to criteria which depend on the decision situation. In the literature, it is well acknowledged that presenting the DM with too many alternatives increases the burden of his or her decision making task.

Different approaches can be undertaken for introducing DM preferences in the process of searching for solutions; a common classification is based on when the DM is consulted: a priori, a posteriori, or interactively during the search. A priori methods use DM preferences to bias the search of optimal solutions towards a preferred region, for example by changing the definition of dominance ([9],[21]), by weighting differently the objectives of the optimization [18], by assigning reference values (goals) and priority levels to them [20], by assuming a utility function describing the DM behavior and interest in the alternative solutions [7]. Interactive methods require the direct intervention of the DM in the optimal solution search, for example simply to stop an iterative trial-and-error search when satisfactory results are reached [6] or more effectively to drive the optimization by ranking and eliminating alternatives based on indicated preference strengths ([4],[7],[15],[16] and [17]) or by bounding DM utility functions by elicited preference information ([3] and [12]); this is to be done while accounting for the fact that the consequences of the alternative solutions may not be completely known, the problem definition may not be exact and the DM preferences may be only partially known and even partially inconsistent. A posteriori methods, on the other hand, apply DM preferences only after the solutions of the Pareto Front are found; the selection task by the DM can be difficult when the Pareto Front contains a large number of solutions. To make the task feasible, only a small number of solutions representative of the Pareto Front should be offered for selection to the DM.

In this work, an a posteriori procedure is proposed for reducing the set of Pareto solutions on a Pareto Front to a small number of representative solutions, according to the DM preferences. First, the set of optimal solutions constituting the Pareto Front and Set is partitioned by subtractive clustering in clusters (here also called "families") of solutions sharing common features [1]. The clustering is based on the geometrical distance between solutions in the objective values space. Then, the representative solution (the "head of the family") is selected for each family (or cluster) on the basis of the DM preferences in order to provide him or her with the most preferred solutions. To this purpose, a scoring system based on a fuzzy preference assignment is developed.

The procedure is applied to a problem concerning the optimization of the test intervals of the components of a

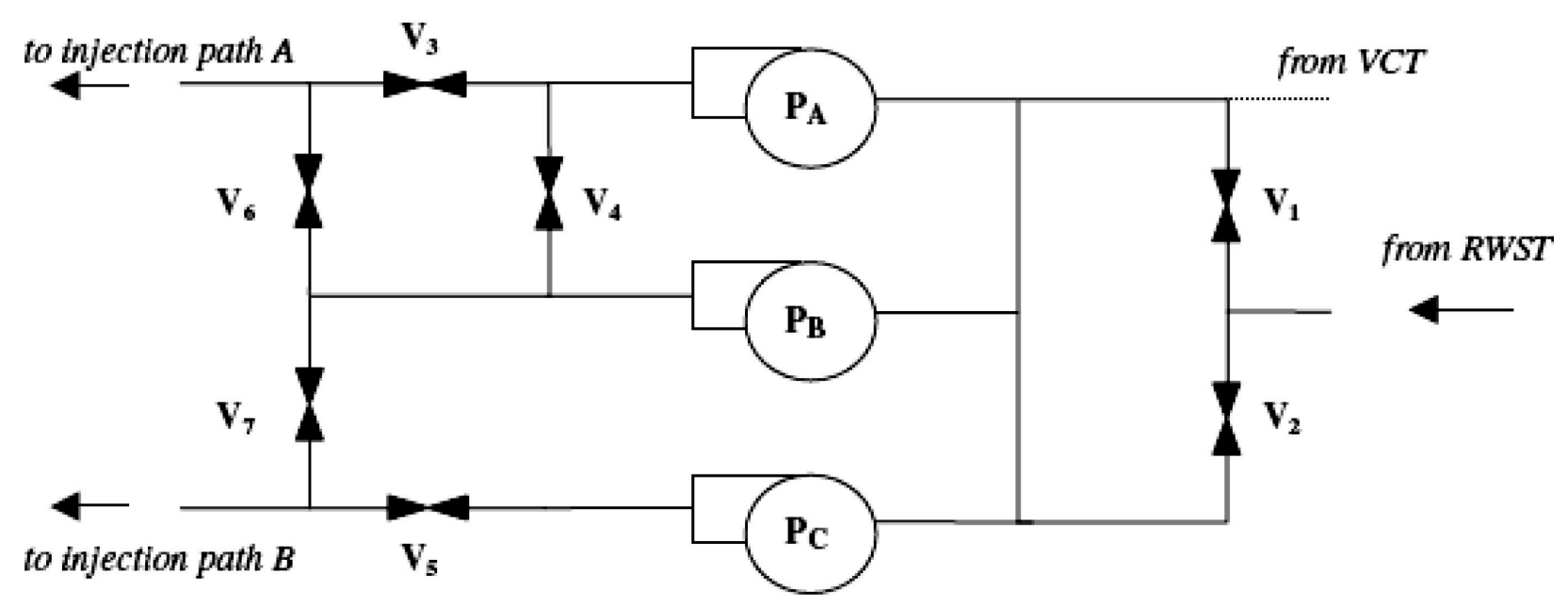

Fig. 1. The Simplified HPIS System (RWST = Radioactive Waste Storage Tank) [2] 
nuclear power plant safety system. The optimization considers three objectives: maximize system availability, minimize cost (from operation \& maintenance and safety issues) and minimize workers exposure time [2]. Level Diagrams [3] are used to graphically represent, analyze and interpret the Pareto Front and Set.

The paper is organized as follows: Section 2 presents the case study; Section 3 contains the analysis of the clustering algorithm and the results of its application; Section 4 presents the selection of the representative heads of the families by fuzzy preference assignment and the application to the case study; Section 5 gives the conclusions that can be drawn from the findings of the work.

\section{OPTIMIZATION OF THE TEST INTERVALS OF THE COMPONENTS OF A NUCLEAR POWER PLANT SAFETY SYSTEM}

The case study here considered is taken from [5] and regards the optimization of the test intervals (TIs) of the high pressure injection system (HPIS) of a pressurized water reactor (PWR), with respect to three objectives: maximize mean system availability, minimize cost and minimize workers time of exposure to radiation.

Figure 1 shows a simplified schematics of a specific HPIS design. The system consists of three pumps and seven valves for a total of $N_{c}=10$ components. During normal reactor operation, one of the three charging pumps draws water from the volume control tank (VCT) in order to maintain a normal level of water in the primary reactor cooling system (RCS) and to provide a small high-pressure flow to the seals of the RCS pumps. Following a small loss of coolant accident (LOCA), the HPIS is required to supply a high pressure flow to the RCS. Moreover, the HPIS can be used to remove heat from the reactor core if the steam generators are completely unavailable. Under normal conditions, the HPIS function is performed by injection through valves $V_{3}$ and $V_{5}$ but, for redundancy, crossover valves $V_{4}, V_{5}$ and $V_{7}$ provide alternative flow paths if some failure were to occur in one of the nominal paths. This stand-by safety system has to be inspected periodically to test its availability. A TI of $2190 \mathrm{~h}$ is specified by the technical specifications (TSs) for both the pumps and the valves. However, there are several restrictions on the maintenance procedures described in the TS, depending on reactor operations.

For this study the following assumptions are made:

1) At least one of the flow paths must be open at all times.

2) If a component is found to have failed during surveillance and testing, it is returned to an as-good-as-new condition through corrective maintenance or replacement.

3) If a component is found to be operable during surveillance and testing, it is returned to an as-goodas-new condition through restorative maintenance.

4) The process of inspection and testing requires a finite time; while the corrective maintenance (or replacement) requires an additional finite time, the restorative maintenance is supposed to be instantaneous.

The $N_{c}$ system components are characterized by their failure rate $\lambda_{h}, h=1, \ldots, N_{c}$, the cost of the yearly inspection $C_{h t, h}$ and corrective maintenance $C_{h c, h}$, the mean downtime due to corrective maintenance $d h$, the mean downtime due to testing $t_{h}$ and their failure on demand probability $\rho_{h}$ (Table 1). They are also divided in three groups characterized by different test strategies; all the components

Table 1. Characteristics of the System Components

\begin{tabular}{|c|c|c|c|c|c|c|c|c|}
\hline $\begin{array}{c}\text { Component } \\
(j)\end{array}$ & $\begin{array}{l}\text { Component } \\
\text { symbol } \\
\text { (Figure 1) }\end{array}$ & $\begin{array}{c}\lambda_{h} \\
\left(\mathrm{~h}^{-1}\right)\end{array}$ & $\begin{array}{l}C_{h t, h} \\
(\$ / h)\end{array}$ & $\begin{array}{l}C_{h c, h} \\
(\$ / \mathrm{h})\end{array}$ & $\begin{array}{l}d h \\
\text { (h) }\end{array}$ & $\begin{array}{l}t_{h} \\
\text { (h) }\end{array}$ & $\begin{array}{l}\rho_{h} \\
\text { (h) }\end{array}$ & $g$ \\
\hline 1 & $V_{1}$ & $5.83 \times 10^{-6}$ & 20 & 15 & 2.6 & 0.75 & $1.82 \times 10^{-4}$ & 1 \\
\hline 2 & $V_{2}$ & $5.83 \times 10^{-6}$ & 20 & 15 & 2.6 & 0.75 & $1.82 \times 10^{-4}$ & 1 \\
\hline 3 & $V_{3}$ & $5.83 \times 10^{-6}$ & 20 & 15 & 2.6 & 0.75 & $1.82 \times 10^{-4}$ & 2 \\
\hline 4 & $V_{4}$ & $5.83 \times 10^{-6}$ & 20 & 15 & 2.6 & 0.75 & $1.82 \times 10^{-4}$ & 3 \\
\hline 5 & $V_{5}$ & $5.83 \times 10^{-6}$ & 20 & 15 & 2.6 & 0.75 & $1.82 \times 10^{-4}$ & 2 \\
\hline 6 & $V_{6}$ & $5.83 \times 10^{-6}$ & 20 & 15 & 2.6 & 0.75 & $1.82 \times 10^{-4}$ & 3 \\
\hline 7 & $V_{7}$ & $5.83 \times 10^{-6}$ & 20 & 15 & 2.6 & 0.75 & $1.82 \times 10^{-4}$ & 3 \\
\hline 8 & $P_{A}$ & $3.89 \times 10^{-6}$ & 20 & 15 & 24 & 4 & $5.3 \times 10^{-4}$ & 2 \\
\hline 9 & $P_{B}$ & $3.89 \times 10^{-6}$ & 20 & 15 & 24 & 4 & $5.3 \times 10^{-4}$ & 2 \\
\hline 10 & $P_{C}$ & $3.89 \times 10^{-6}$ & 20 & 15 & 24 & 4 & $5.3 \times 10^{-4}$ & 2 \\
\hline
\end{tabular}


belonging to the same group undergo testing with the same periodicity $T^{g}$, with $g=1,2,3$, i.e., they all have the same test interval.

Any solution to the optimization problem can be encoded using the following array $\theta$ of decision variables:

$$
\theta=\left[\begin{array}{lll}
T^{1} & T^{2} & T^{3}
\end{array}\right]
$$

Assuming a mission time (TM) of one year $(8760 \mathrm{~h})$, the range of variability of the three TIs is $[1,8760] \mathrm{h}$.

The search for the optimal test intervals is driven by the following three objective functions $J_{i}(\theta), i=1, \ldots ., 3$ :

Availability:

$$
\max _{\theta} J_{1}(\theta)=\max _{\theta}\left[\left(1-\sum_{v=1}^{N_{M C S}} \sum_{h=1}^{n_{v}} \bar{u}_{h}^{v}(\theta)\right)\right]
$$

Cost:

$$
\min _{\theta} J_{2}(\theta)=\min _{\theta}\left[C_{\text {accident }}(\theta)+\sum_{h=1}^{N_{c}} C_{S \& M, h}(\theta)\right]
$$

\section{Exposure Time:}

$$
\min _{\theta} J_{3}(\theta)=\min _{\theta}\left[\sum_{h=1}^{N_{c}} E T_{h}(\theta)\right]
$$

The HPIS mean unavailability $\bar{U}_{H P I S}$ has been computed from the fault tree for the top event "no flow out of both injection paths A and B"; the Boolean reduction of the corresponding structure function allows us to determine the $N_{M C S}$ system minimal cut sets (MCS) [5]. Then, the system mean unavailability can be expressed as in the argument of the maximization (2) where $n_{v}$ is the number of basic events in the $v$-th minimal cut set and $\bar{u}_{h}^{v}$ is the mean unavailability of the $h$-th component making up the $v$-th MCS, $h=1, \ldots, n_{v}[8]$ :

$$
\bar{u}_{h}^{v}=\rho_{h}+\frac{1}{2} \lambda_{h} \tau_{h}+\left(\rho_{h}+\lambda_{h} \tau_{h}\right) \frac{d_{h}}{\tau_{h}}+\frac{t_{h}}{\tau_{h}}+\gamma_{0}
$$

where $\gamma_{0}$ is the probability of human error. The simple expression in (5) is valid for $\rho_{h}<0.1$ and $\lambda_{h} \tau_{h}<0.1$, which are reasonable assumptions when considering safety systems.

The cost objective $C$ is made up of two major

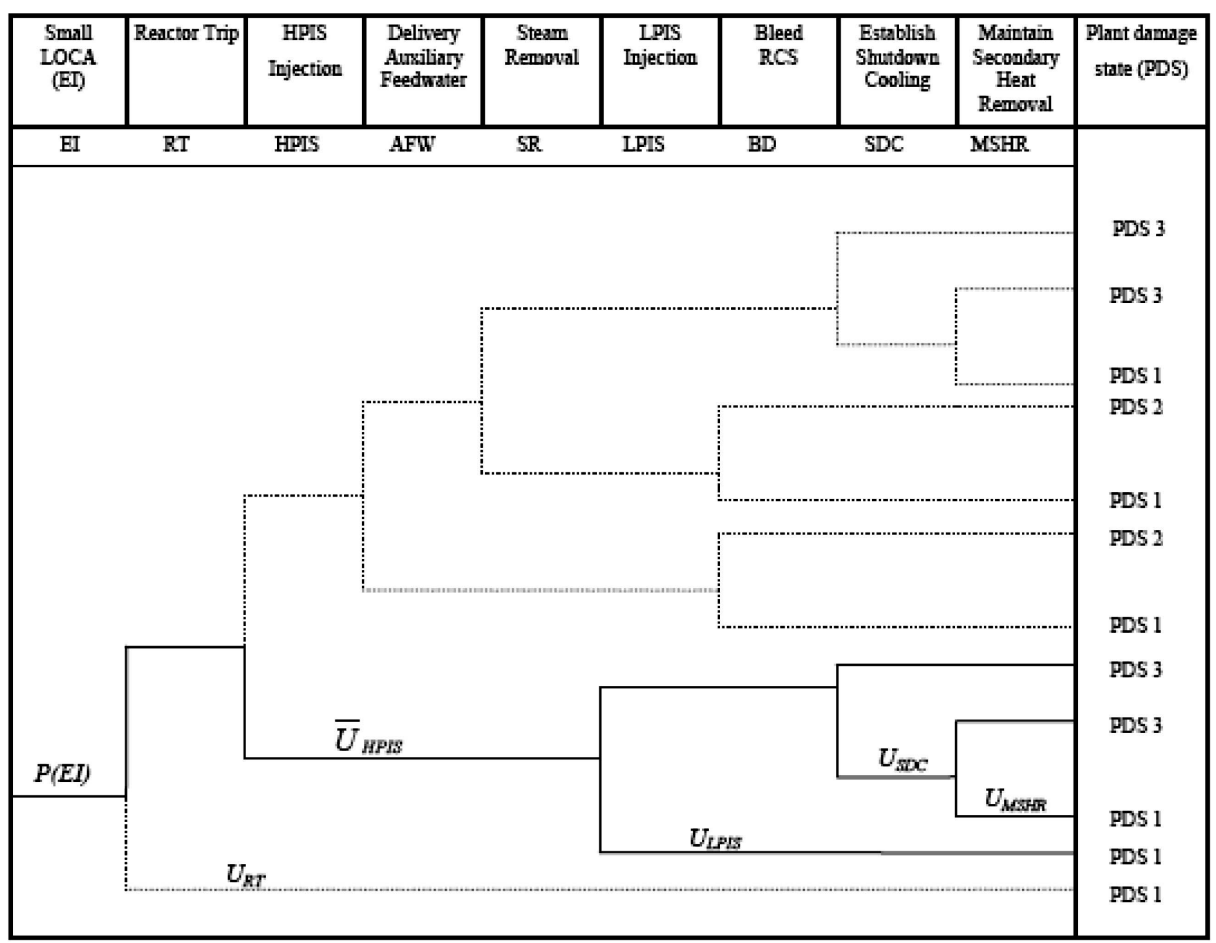

Fig. 2. Small LOCA Event Tree [19] 
contributions: $C_{S \& M}$ the cost associated with the operation of surveillance and maintenance $(\mathrm{S} \& \mathrm{M})$ and $C_{\text {accident }}$ the cost associated with consequences of accidents occurring at the plant.

For a given component $h$, the S\&M cost is computed on the basis of the yearly inspection and corrective maintenance costs. For a given mission time, TM, the number of inspections performed on component $h$ are $\frac{T M}{\tau_{h}}$; of these, on average, a fraction equal to $\left(\rho_{h}+\lambda_{h} \tau_{h}\right)$ demands also a corrective maintenance action of duration $d_{h}$; thus, the S\&M costs amount to

$$
C_{S \& M, h}(\theta)=C_{h t, h} \frac{T M}{\tau_{h}} t_{h}+C_{h c, h}\left(\rho_{h}+\lambda_{h} \tau_{h}\right) \frac{T M}{\tau_{h}} d_{h}, h=1, \ldots ., N_{c}(6)
$$

Concerning the accident cost contribution, it is intended to measure the costs associated with damages of accidents that are not mitigated due to the HPIS failing to intervene. A proper analysis of such costs implies that we account for the probability of the corresponding accident sequences; for simplicity, but with no loss of generality, consideration here is limited to only the accident sequences relative to a small LOCA event tree of literature (Figure 2) [21].

The accident sequences considered for the quantification of the accident costs are those which involve the failure of the HPIS (thick lines in Figure 2), so that the possible Plant Damage States (PDS) are PDS1 and PDS3. Thus,

$$
\left\{\begin{array}{c}
C_{\text {accident }}=C_{1}+C_{3} \\
C_{1}=P(E I) \cdot\left(1-U_{R T}\right) \cdot \bar{U}_{H P I S} \cdot\left\{U_{L P I S}+\left(1-U_{L P I S}\right) \cdot U_{S D C} \cdot U_{M S H R}\right\} \cdot C_{P D S 1}(7) \\
C_{3}=P(E I) \cdot\left(1-U_{R T}\right) \cdot \bar{U}_{H P I S} \cdot\left(1-U_{L P I S}\right) \cdot\left\{\left(1-U_{M S H R}\right) \cdot U_{S D C}+\left(1-U_{S D C}\right)\right\} \cdot C_{P D S 3}
\end{array}\right.
$$

where $C_{1}$ and $C_{3}$ are the total costs associated with accident sequences leading to damaging states 1 and 3 , respectively. These costs depend on the initiating event frequency $P(E I)$ and on the unavailability values $U_{i}$ of the safety systems which ought to intervene along the various sequences: these values are taken from the literature ([10] and [19]) for all systems except for the SDC (ShutDown Cooling) and MSHR (Maintain Secondary Heat Removal), which were not available and were arbitrarily assumed of the same order of magnitude of the other safety systems, and for the HPIS for which the unavailability $\overline{\mathrm{U}}_{H P I S}$ is calculated from (2) and (5) and it depends on the TIs of the components. Finally, for the values of $C_{P D S 1}$ and $C_{P D S 3}$, the accident costs for PDS1 and PDS3, respectively, are taken as the mean values of the uniform distributions given in [19].

Table 2 summarizes the input data.

During testing operations, the technicians may be subjected to radiation exposure. With reference to the ICRP recommendation $n^{\circ} 60$ [11], based on the well known ALARA (As Low As Reasonably Achievable) and limitdose principles, the dose received by workers should be minimized. Assuming a constant exposure rate, the minimization of the dose is equivalent to that of the exposure time (ET), and the exposure time due to a single component $h$ can be computed as

$$
E T_{h}(\theta)=\frac{T M}{\tau_{h}} t_{h}+\left(\rho_{h}+\lambda_{h} \tau_{h}\right) \frac{T M}{\tau_{h}} d_{h}, h=1, \ldots, N_{c}
$$

The multiobjective optimization problem (2)-(4) has been solved using the MATLAB genetic algorithm toolbox, with a population size of 100 individuals and a stopping criterion of 500 generations, as indicated in [5]. The resulting Pareto Set $(\Theta)$ is made of 100 points, and the corresponding Pareto Front is shown in Figure 3 in the objective functions space.

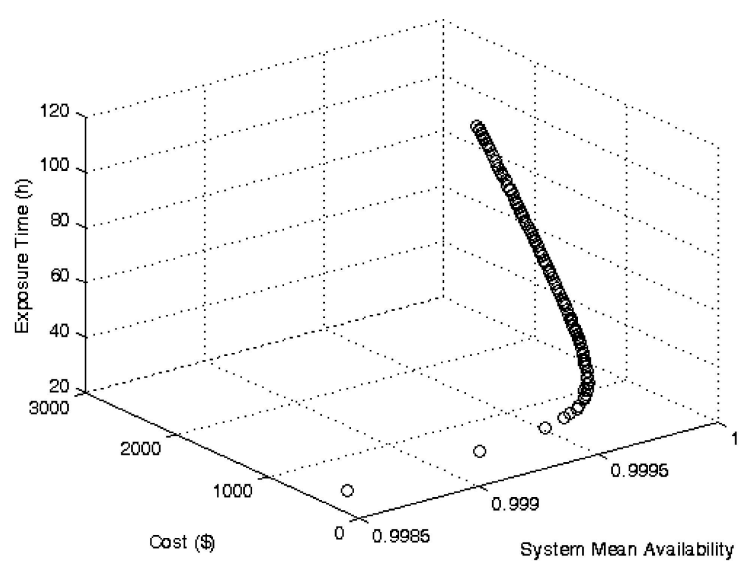

Fig. 3. Pareto Front, in the Objective Functions Space, Obtained by the MATLAB Genetic Algorithm

Table 2. Accident Cost Input Data [2]

\begin{tabular}{c|c|c|c|c|c|c}
\hline $\begin{array}{c}P(E I) \\
\left(\mathrm{y}^{-1}\right)\end{array}$ & $\begin{array}{c}U_{R T} \\
\left(\mathrm{y}^{-1}\right)\end{array}$ & $\begin{array}{c}U_{L P I S} \\
\left(\mathrm{y}^{-1}\right)\end{array}$ & $\begin{array}{c}U_{S D C} \\
\left(\mathrm{y}^{-1}\right)\end{array}$ & $\begin{array}{c}U_{M S H R} \\
\left(\mathrm{y}^{-1}\right)\end{array}$ & $\begin{array}{c}C_{P D S 1} \\
(\$ \times e v e n t)\end{array}$ & $\begin{array}{c}C_{P D S 2} \\
(\$ \times e v e n t)\end{array}$ \\
\hline $2.43 \times 10^{-5}$ & $3.6 \times 10^{-5}$ & $9 \times 10^{-3}$ & $5 \times 10^{-3}$ & $5 \times 10^{-3}$ & $2.1765 \times 10^{9}$ & $1.375 \times 10^{8}$ \\
\hline
\end{tabular}




\section{CLUSTERING OF THE PARETO FRONT}

\subsection{Subtractive Clustering Algorithm}

The first step of the procedure for reducing the number of solutions to present to the DM is to group the solutions of the Pareto Front in a number $K$ of families of solutions sharing similar characteristics. In this work, subtractive clustering [2] is used to identify the families of similar solutions (clusters) $F^{j}, j=1, \ldots ., K$, in the objective functions space. The clustering is performed on the basis of the geometrical distance between solutions, i.e., in this case, the geometrical distance between objective function values.

Subtractive clustering has been chosen over other methods like the k-means and fuzzy c-means, because it does not require the solution of a minimization problem of an objective function nor any random initialization so that the results are independent of the initial cluster centers or membership function choice; finally, different from the $\mathrm{k}$-means and the fuzzy c-means, the cluster centers $\underline{J}_{\text {norm }}^{j}$, $j=1, \ldots ., K$ are objective values vectors corresponding to existing solutions in the Pareto Front and Set and thus can be used directly as representative solutions on the reduced Pareto Front.

Let us consider a Pareto Set $\Theta$ made of $n$ solutions; to the $i$-th solution $\theta^{i}(i=1, \ldots, n)$ corresponds a vector of objective values

$$
\underline{J}\left(\theta^{i}\right)=\left(\begin{array}{llll}
J_{1}\left(\theta^{i}\right) & \left.J_{2}\left(\theta^{i}\right) \quad \ldots \quad J_{\text {Nobj }}\left(\theta^{i}\right)\right)
\end{array}\right)
$$

where $N_{o b j}$ is the number of objective functions of the optimization problem. Since the objective functions are usually given in different units and scales, their values are normalized with respect to the minimum value in the Pareto Front:

$$
\begin{gathered}
\underline{J}_{\text {norm }}\left(\theta^{i}\right)=\left(J_{1, \text { norm }}\left(\theta^{i}\right) \quad J_{2, \text { norm }}\left(\theta^{i}\right) \quad \ldots \quad J_{\text {Nobj,norm }}\left(\theta^{i}\right)\right) \\
J_{s, \text { orm }}\left(\theta^{i}\right)=\frac{J_{s}\left(\theta^{i}\right)-J_{s, \min }}{J_{s, \max }-J_{s, \min }}, s=1, \ldots ., N_{o b j}
\end{gathered}
$$

where

$$
J_{s, \min }=\min _{i} J_{s}\left(\theta^{i}\right) \text { and } J_{s, \max }=\max _{i} J_{s}\left(\theta^{i}\right) .
$$

The subtractive clustering examines the optimal solutions along the Pareto Front to define the cluster centers according to the density of surrounding solutions.
The flowchart of the subtractive clustering algorithm is given in Figure 4.

Given the Pareto Front made of $n$ normalized solutions $\underline{J}_{\text {norm }}\left(\theta^{i}\right)$, the algorithm starts by calculating the following potential $P\left(\underline{J}_{\text {norm }}\left(\theta^{i}\right)\right), i=1,2, \ldots, n$ :

$$
P\left(\underline{J}_{\text {norm }}\left(\theta^{i}\right)\right)=\sum_{l=1}^{n} e^{-\alpha \mid \underline{n}_{\text {nom }}\left(\theta^{i}\right) \underline{-n}_{\text {nom }}\left(\theta^{i}\right)^{2}}, \alpha=\frac{4}{r_{a}^{2}}
$$

where $r_{a} \in[0,1]$ is an input parameter called cluster radius, which indicates a cluster center's range of influence in each of the data dimensions, assuming the solution space is a unit hyperbox; a solution outside the cluster radius $r a$ of the cluster center has a small influence on the potential. The choice of the parameter $r_{a}$ determines the number $K$ of clusters that will be identified: a large value of $r a$ gives only a few large clusters; usually this parameter is taken smaller than 0.5 .

After the potentials of all solutions have been computed, the first cluster center $\underline{J}_{n o r m}^{1}$ is selected as the solution with the highest potential value $P\left(\underline{J}_{\text {norm }}^{1}\right)$. All the other $n-1$ solutions potentials $P\left(\underline{J}_{\text {norm }}\left(\theta^{i}\right)\right)$ are corrected by subtracting the potential $P\left(\underline{J}_{\text {norm }}^{1}\right)$ multiplied by a factor which considers the distance between the $i$-th solution and the

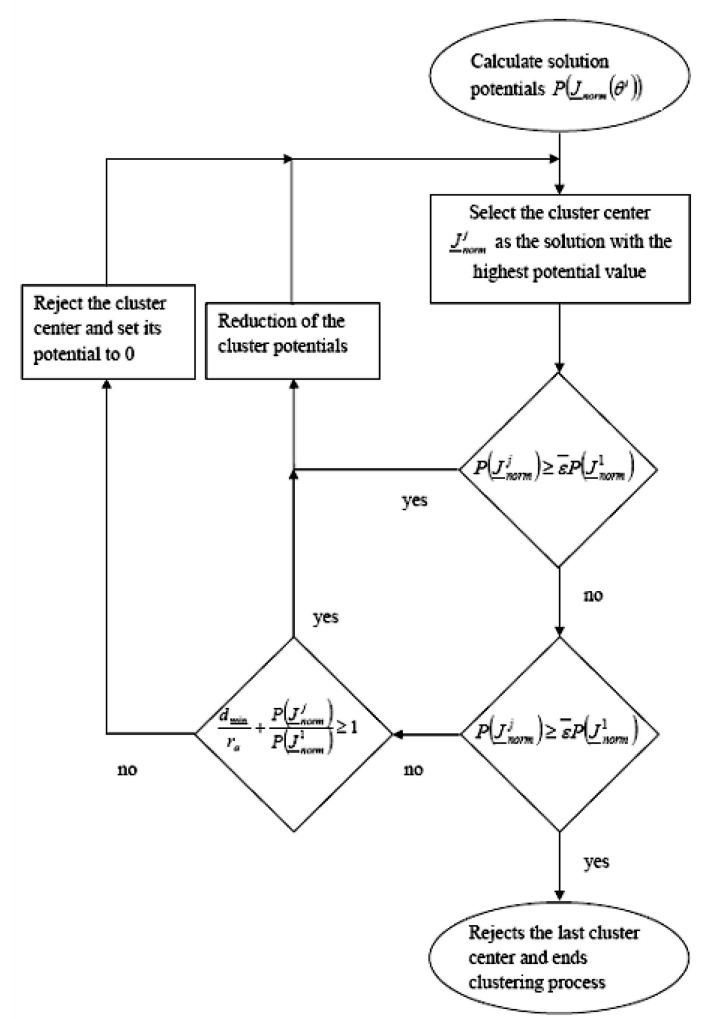

Fig. 4. Flowchart of the Subtractive Clustering Algorithm 
first cluster center:

$$
\begin{gathered}
P\left(\underline{J}_{\text {norm }}\left(\theta^{i}\right)\right)=P\left(\underline{J}_{\text {norm }}\left(\theta^{i}\right)\right)-P\left(\underline{J}_{\text {norm }}^{1}\right) e^{-\beta\left|\underline{I n o r m}_{\text {norm }}\left(\theta^{i}\right)-\underline{-}_{\text {norm }}^{1}\right|^{2}}, \\
\beta=\frac{4}{r_{b}^{2}} \text { and } r_{b}=q r_{a}
\end{gathered}
$$

where $q$ is an input parameter called the squash factor, which indicates the neighborhood with a measurable reduction of potential expressed as a fraction of the cluster radius; a value of 2 of the squash factor indicates that the clusters found are far from each other.

At this stage, from the $n-1$ remaining solutions, the one with the highest value of the reduced potential is chosen as the second cluster center $\underline{J}_{n o r m}^{2}$.

Generally, for the $j$-th cluster center found $\underline{j}_{\text {norm }}^{j}, j=1, \ldots$, $K$, the potentials are reduced as follows:

$$
P\left(\underline{J}_{\text {norm }}\left(\theta^{i}\right)\right)=P\left(\underline{J}_{\text {norm }}\left(\theta^{i}\right)\right)-P\left(\underline{J}_{\text {norm }}^{j}\right) e^{-\beta\left|I_{\text {norm }}\left(\theta^{i}\right)-\underline{-}_{\text {nom }}^{j}\right|^{2}} .
$$

Two other input parameters are introduced: the accept ratio $\bar{\varepsilon}$ and the reject ratio $\underline{\varepsilon}$, which are respectively the fraction of the potential of the first cluster center above or below which another solution is accepted or rejected as a cluster center. Then, the process of finding new cluster centers and reducing the potential according to (14), is repeated according to the following criteria: if

$$
P\left(\underline{J}_{\text {norm }}^{j}\right) \geq \bar{\varepsilon} P\left(\underline{J}_{\text {norm }}^{1}\right)
$$

the cluster center $\underline{J}_{n o r m}^{j}$ is accepted and then the potential of the other solutions are further reduced as in (21). If

$$
P\left(\underline{J}_{\text {norm }}^{j}\right) \leq \underline{\varepsilon} P\left(\underline{J}_{\text {norm }}^{1}\right)
$$

the cluster center $\underline{J}_{n o r m}^{j}$ is rejected and the clustering process terminates. If neither (15) nor (16) are satisfied, then the acceptance criterion becomes

$$
\frac{d_{\min }}{r_{a}}+\frac{P\left(\underline{J}_{\text {norm }}^{j}\right)}{P\left(\underline{J}_{\text {norm }}^{1}\right)} \geq 1
$$

where $d_{\text {min }}$ is defined as

$$
d_{\min }=\min _{h}\|\|_{\text {norm }}^{j}-\underline{J}_{\text {norm }}^{h} \|_{2} \text { with } h=1, \ldots ., j-1 .
$$

Finally, when all the cluster centers have been computed, a membership function matrix $\mu$ is found using the standard Gaussian distribution:

$$
\mu_{j, i}=e^{-\alpha\left|\underline{J}_{\text {norm }}\left(\theta^{i}\right)-\underline{-}_{\text {norm }}^{j}\right|^{2}}
$$

Since the membership function (18) is computed as a negative power of the distance between the solution $\underline{J}_{n o r m}\left(\theta^{i}\right)$ and the cluster centers $\underline{J}_{\text {norm }}^{j}$, the smaller the distance, the higher the membership function.

The subtractive clustering algorithm allows us to avoid the problem of objective function minimization; there is also no random initialization so that the results are not dependent on the initial cluster centers or membership function choice; finally, the cluster centers $\underline{J}_{n o r m}^{j}, j=1, \ldots ., K$, are objective values vectors corresponding to existing solutions in the Pareto Front and Set and thus could be used directly as representative solutions on the reduced Pareto Front.

\subsection{Clustering the Pareto Front of the case study of Section 2}

The algorithm presented in Section 3.1 has been applied for clustering the Pareto Front solutions of the case study presented in Section 2.

The default input parameters are given in Table 3. They were set after we analyzed the results of the clustering obtained with different values of the parameters.

The cluster radius $r a$, which influences directly the number of clusters $K$ obtained by the subtractive clustering algorithm, is an important parameter in the Pareto Front reduction procedure because it determines how many families (clusters), and consequently representative solutions, the Pareto Front is reduced to. To optimally set it, the so-called global silhouette value is used to evaluate the quality of the clustering allocation ([13] and [14]). For any cluster partition of the Pareto Front, a global silhouette index, $G S$, is computed as follows:

$$
G S=\frac{1}{K} \sum_{j=1}^{K} S_{j}
$$

where $S_{j}$ is the cluster silhouette of the $j$-th cluster $F^{j}$ indicating the heterogeneity and isolation properties of the

Table 3. Default Input Parameters for the Subtractive Clustering Algorithm

\begin{tabular}{c|c|c}
\hline$q$ & $\bar{\varepsilon}$ & $\underline{\varepsilon}$ \\
\hline 1.25 & 0.5 & 0.15 \\
\hline
\end{tabular}


cluster; it is computed as the average value of the silhouette widths $s(i)$ of the solutions in the cluster $j$, defined as

$$
s(i)=\frac{b(i)-a(i)}{\max \{a(i), b(i)\}}
$$

where, $a(i)$ is the average distance from the $i$-th solution of all the other solutions in the cluster, and $b(i)$ is the average distance from the $i$ - $t h$ solution of all the solutions in the nearest neighbor cluster identified as the cluster with the minimum average distance of all its solutions from the $i$-th solution.

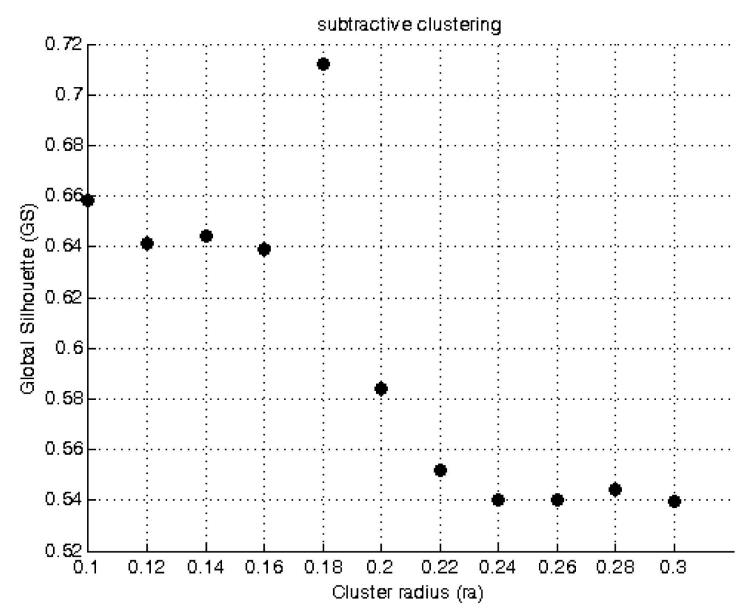

Fig. 5. GS for Different Cluster Radius Values
The value of $s(i)$ ranges between +1 and -1 ; a value of +1 indicates solutions that are very distant from neighboring clusters; a value of 0 indicates solutions that are not distinctly in one cluster or another; a value of -1 indicates solutions that are probably assigned to the wrong cluster.

The maximum global silhouette can be used to define the optimal number of clusters [13].

The values of the global silhouette obtained for different values of $r_{a}$ with default settings are given in Figure 5: the highest value of the global silhouette $(0.71)$ is reached for a cluster radius value $r_{a}=0.18$, which results in $K=9$ clusters, and the other parameters are set as in the default configuration of Table 3.

The results of the subtractive clustering are showed in Figure 6.

\section{CLUSTER REPRESENTATIVE SOLUTION SELECTION}

Each family of solutions $F^{j}, j=1, \ldots, K$, obtained by the subtractive clustering algorithm contains a number $n^{j}$ of solutions of similar characteristics in the objective functions space and whose best representative solution, the head of the family $\underline{H}^{j}=\left(H_{1}^{j} \ldots . H_{S}^{j} \ldots . H_{N_{o b} j}^{j}\right)$, needs to be found, accounting for the DM specific requirements. The DM would then be provided with only this reduced number $K$ of solutions $\underline{H}^{j}, j=1, \ldots ., K$, which best represent the Pareto Front under analysis, according to his or her preferences, and from which to select the final solution of the optimization problem.

The clusters solutions can be visualized using Level

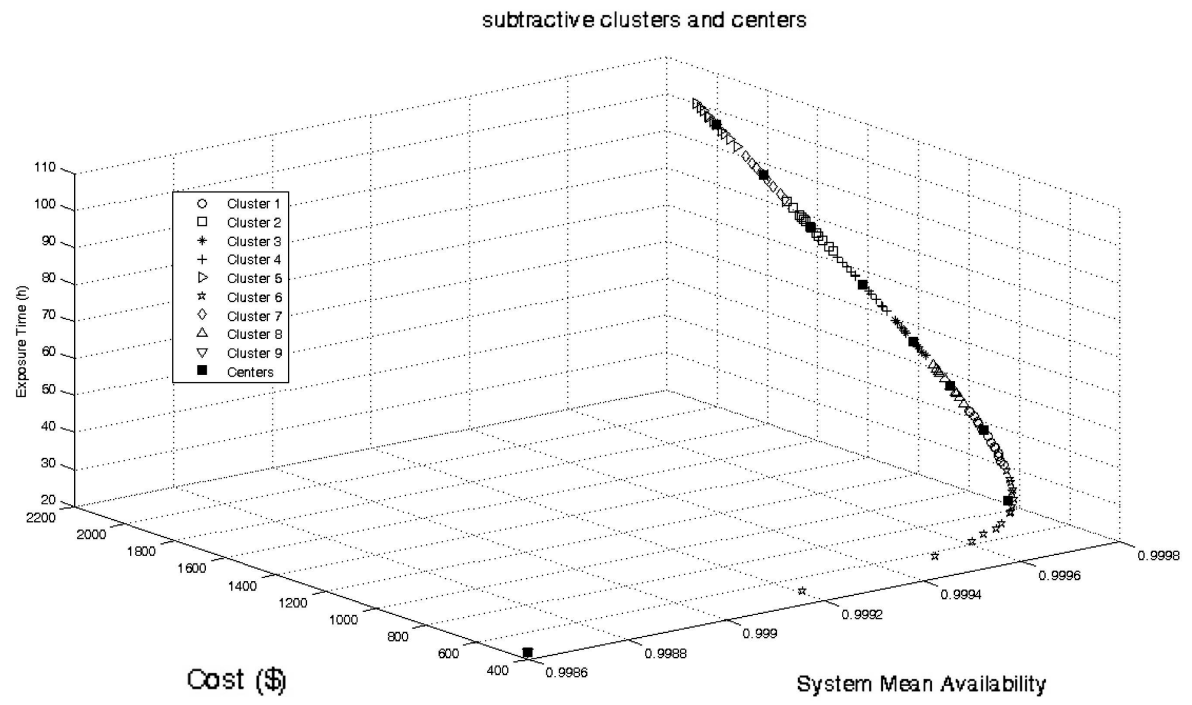

Fig. 6. Solutions Clusters and Centers Obtained with the Subtractive Clustering Algorithm in the Objective Function Space of the Test Intervals Optimization Case Study 
Diagrams [1], which account for the distance of the Pareto Front and Set solutions from the ideal solution, optimal with respect to all the objectives simultaneously. Considering a multiobjective problem with $l$ objectives to be minimized and $m$ maximized (such that $N_{o b j}=l+m$ ), $n$ solutions in the Pareto Set and indicating by $J\left(\theta^{i}\right)=\left(J_{1}\left(\theta^{i}\right) \ldots . J_{s}\left(\theta^{i}\right) \ldots . J_{\text {Nobj }}\right.$ $\left(\theta^{i}\right)$ ) the objective functions values vector corresponding to the solution $\theta^{i}, i=1, \ldots ., n$, each objective value $J_{s}\left(\theta^{i}\right), s=1, \ldots$. , $N_{o b j}$ can be normalized with respect to its minimum and maximum values $\left(\boldsymbol{J}_{s}^{\min }\right.$ and $\left.\boldsymbol{J}_{s}^{\max }\right)$ on the Pareto Front [1]:

$$
\overline{J_{s}}\left(\theta^{i}\right)=\frac{J_{s}\left(\theta^{i}\right)-J_{s}^{\min }}{J_{s}^{\max }-J_{s}^{\min }} \quad s=1, \ldots ., l
$$

and

$$
\overline{J_{s}}\left(\theta^{i}\right)=\frac{J_{s}^{\max }-J_{s}\left(\theta^{i}\right)}{J_{s}^{\max }-J_{s}^{\min }} \quad s=1, \ldots, m
$$

so that now,

$$
0 \leq \overline{J_{s}}\left(\theta^{i}\right) \leq 1 \quad s=1, \ldots ., N_{o b j},
$$

where

- $\overline{J_{s}}\left(\theta^{i}\right)=0$ means that solution $\theta^{i}$ has the best value for the $s$-th objective

- $\overline{J_{s}}\left(\theta^{i}\right)=1$ means that solution $\theta^{i}$ has the worst value for the $s$-th objective.

To evaluate the distance to the ideal point

$$
\theta^{*}: \overline{J_{s}}\left(\theta^{i}\right)=0 \forall s=1, \ldots, N_{o b j},
$$

a suitable norm must be introduced. Different norms can give different views on the characteristics of the Pareto Front and Set [5]. In this paper, the norm considered is the following 1-norm:

1-norm :

$$
\left\|\underline{J}\left(\theta^{i}\right)\right\|_{1}=\sum_{s=1}^{N o b j} \overline{J_{s}}\left(\theta^{i}\right) \text {, with } 0 \leq\left\|J\left(\theta^{i}\right)\right\|_{1} \leq s
$$

We chose this norm because it takes into account all the objectives and thus can be used to analyze the performance of the solution in an all-around way. The plot of Level Diagrams is then done as follows: each objective $J_{s}, s=1, \ldots, N_{o b j}$ is plotted separately; the $\mathrm{X}$ axis corresponds to the objective in physical units of measurement, while the $\mathrm{Y}$ axis corresponds, for all the graphs, to the value $\left\|\underline{J}\left(\theta^{i}\right)\right\|_{1}$. This means that all the plots are synchronized with respect to the $\mathrm{Y}$ axis, i.e., all the information for a single solution of the Pareto Front will be plotted at the same level of the $\mathrm{Y}$ axis.

The clustered Pareto Front of the case study of Section 2 is represented by Level Diagrams in Figure 7.

When analyzing the Pareto Front, the DM applies his
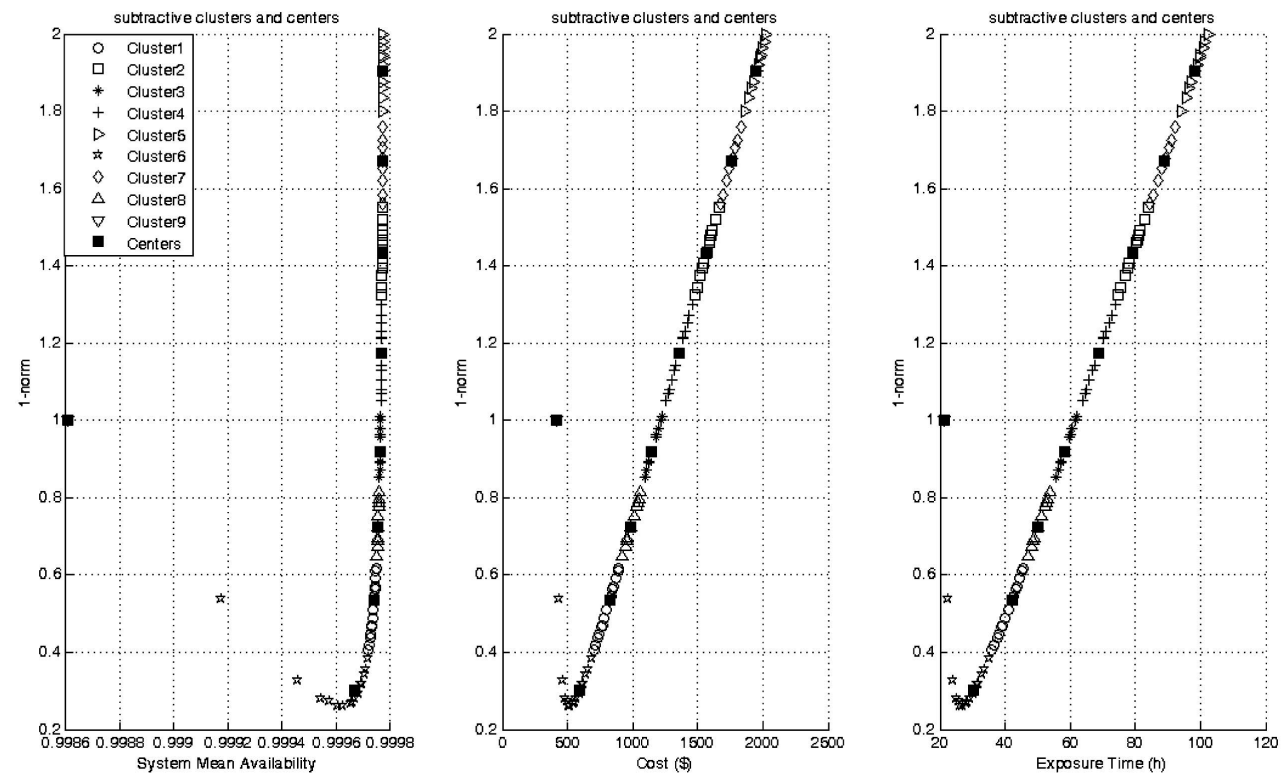

Fig. 7. Level Diagrams Representation of the Pareto Front of the Test Intervals Optimization Case Study (Section 2) 
or her preferences on the objective functions values to identify the best solutions according to these preferences.

The solutions most relevant for the DM are those which are best with respect to the DM preferences.

In this work, the assignment of the DM preferences to the solutions is done by assigning objective values thresholds, which define different classes of merit. The objective values thresholds are given in a preference matrix $P\left(N_{o b j} \times C\right)$, where $C$ is the number of objective functions thresholds used for the classification, defining $C+1$ preference classes as follows [1]:

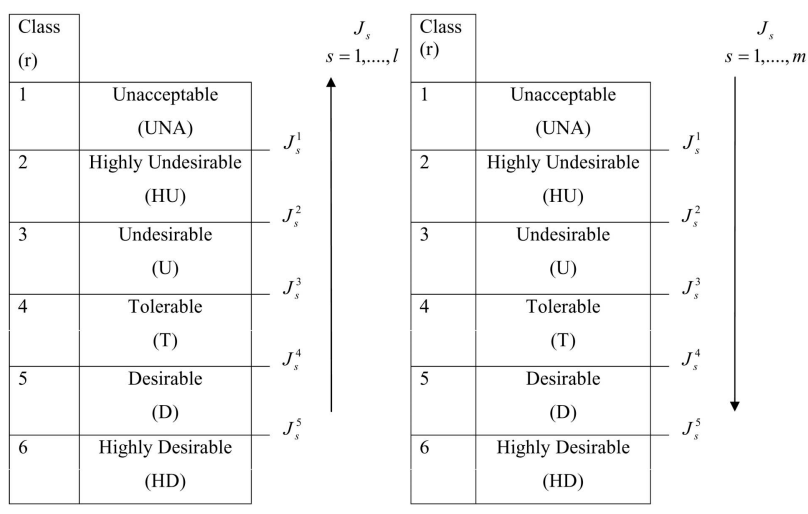

where $J_{s}^{z}, Z=1, \ldots, 5$ are the thresholds values of the $s$-th objective, $l$ and $m$ are the number of objectives to be minimized and maximized, respectively.

The fuzzy scoring procedure introduced by the authors in [22] is then applied: each preference class is assigned a score $s v(r)[1], r=1, \ldots, C+1$, such that

$$
s v(C+1)=0 ; s v(r)=N_{o b j} \cdot s v(r+1)+1, \text { for } r=C, \ldots ., 1
$$

and each objective value $J_{s}\left(\theta^{i}\right), i=1, \ldots, n$ and $s=1, \ldots, N_{o b j}$ is assigned a membership function $\mu_{A_{s}^{r}}\left(J_{s}\left(\theta^{i}\right)\right)$, which represents the degree with which $J_{s}\left(\theta^{i}\right)$ is compatible with the fact of belonging to the $r$ - $t h$ preference class, $r=1, \ldots ., C+1$.

A vector of $C+1=6$ membership functions is then defined for each objective $J_{s}$ :

$$
\begin{gathered}
\underline{\mu}\left(J_{s}\left(\theta^{i}\right)\right)=\left(\mu_{A_{s}^{1}}\left(J_{s}\left(\theta^{i}\right)\right) \quad \mu_{A_{s}^{2}}\left(J_{s}\left(\theta^{i}\right)\right) \quad \mu_{A_{s}^{3}}\left(J_{s}\left(\theta^{i}\right)\right)\right. \\
\left.\mu_{A_{s}^{4}}\left(J_{s}\left(\theta^{i}\right)\right) \quad \mu_{A_{s}^{s}}\left(J_{s}\left(\theta^{i}\right)\right) \quad \mu_{A_{s}^{6}}\left(J_{s}\left(\theta^{i}\right)\right)\right), \\
i=1, \ldots ., n, s=1, \ldots, N_{o b j} .
\end{gathered}
$$

The membership-weighted score of each individual objective is then computed; given the scoring vector $\underline{s v}=(s v(1) s v(2) \ldots s v(C+1))$, whose components are defined in (27), and the membership functions vector $\underline{\mu}\left(J_{s}\left(\theta^{i}\right)\right)$ in (28) for the $i$-th solution and $s$-th objective function, the score $s v_{s}^{i}$ of the individual objective $J_{s}$ is obtained by weighting the score $s v\left(r_{s}\right)$ of each class $r_{s}$ the objective belongs to, by the respective membership function value $\mu_{A_{s}^{r_{s}}}\left(J_{s}\left(\theta^{i}\right)\right), r_{s}=1, \ldots, 6$, and then summing the 6 resulting terms. This can be formulated in terms of the scalar product of the vectors $\underline{\mu}\left(J_{s}^{i}\right)$ and $\underline{s v}$ as follows:

$$
s v_{s}^{i}=\frac{\left\langle\underline{\mu}\left(J_{s}\left(\theta^{i}\right)\right), \underline{s v}\right\rangle}{\sum_{r_{s}=1}^{6} \mu_{A_{s}^{r_{s}}}\left(J_{s}\left(\theta^{i}\right)\right)}, i=1, \ldots ., n \text { and } s=1, \ldots ., N_{o b j},
$$

where the denominator serves as the normalization factor.

Then, the score $S\left(\underline{J}\left(\theta^{i}\right)\right)$ of the $i$-th solution is the sum of the scores of the individual objectives

\begin{tabular}{|c|c|c|c|c|c|}
\hline & $J_{S}^{1}$ & $J_{S}^{2}$ & $J_{S}^{3}$ & $J_{S}^{4}$ & $J_{S}^{5}$ \\
\hline $\begin{array}{c}J_{1} \\
\text { (mean availability) }\end{array}$ & 0.9975 & 0.998 & 0.9985 & 0.999 & 0.9995 \\
\hline $\begin{array}{c}J_{2} \\
(\operatorname{cost}(\$))\end{array}$ & 900 & 800 & 700 & 600 & 500 \\
\hline $\begin{array}{c}J_{3} \\
(\text { exposure time }(\mathrm{h}))\end{array}$ & 60 & 50 & 45 & 40 & 30 \\
\hline
\end{tabular}

$$
S\left(\underline{J}\left(\theta^{i}\right)\right)=\sum_{s=1}^{N_{o b j}} s v_{s}^{i}, i=1, \ldots, n
$$

and the lowest score is taken as the most preferred solution.

Table 4. Preference Threshold Matrix Pfor the Test Intervals Optimization Case Study of Section 2 

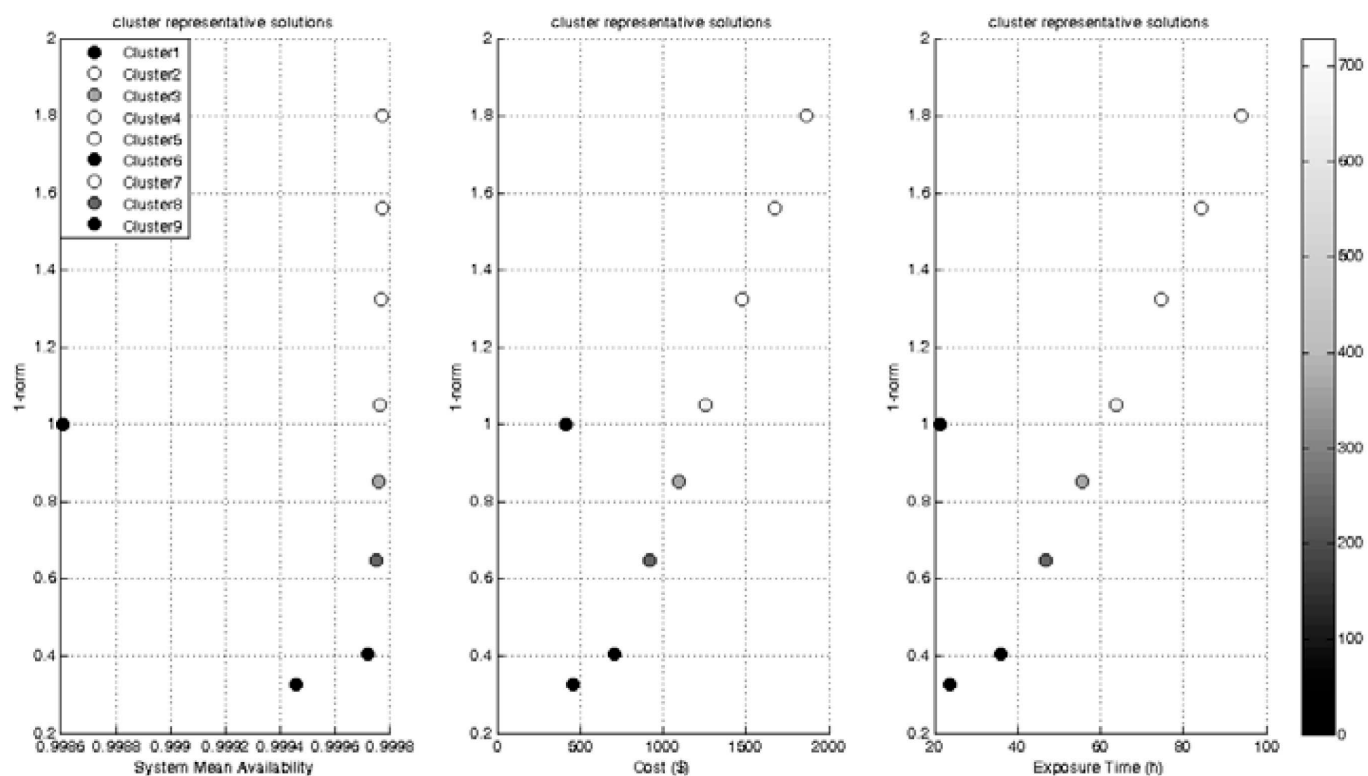

Fig. 8. Level Diagrams Representation of the Family Representative Solutions with the Lowest Score $\mathrm{S}\left(H^{j}\right)$ for the Case Study of Test Intervals Optimization (Section 2)

According to this fuzzy scoring procedure, the head $\underline{H}^{j}$ of the generic family $F^{j}, j=1, \ldots ., K$ is chosen as the solution in $F^{j}$ with lowest scores $S\left(\underline{J}\left(\theta^{i}\right)\right)$ :

$$
S\left(\underline{H}^{j}\right)=\min S\left(\underline{J}\left(\theta^{i}\right)_{k}\right), k=1, \ldots ., n^{j} \text { and } j=1, \ldots ., K
$$

For illustration purposes, let us introduce the arbitrary preference matrix $P$ of Table 4 for the test intervals optimization case study of Section 2.

The resulting family representative solutions are visualized in Figure 8 by Level Diagrams combined with a colouring method to represent the different scores $S\left(H^{j}\right)$ [1]. It can be seen that the Pareto Front shape and coverage is maintained after reduction.

Finally, the proposed procedure for reducing the solutions of the Pareto Front and Set for presentation to the DM is summarized in Figure 9.

\section{CONCLUSIONS}

The algorithms of multiobjective optimization identify a Pareto Set of non-dominated solutions among which the DM has to select the preferred ones. In practical applications, the selection is difficult because the set of non-dominated solutions can be quite large.

In this work, an a posteriori procedure for reducing the set of solutions for presentation to the DM is
Pareto Front and Set of $n$ solutions

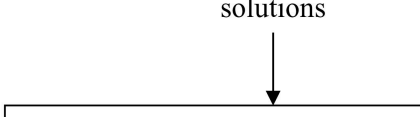

Subtractive clustering algorithm in the objective functions space (Pareto Front)

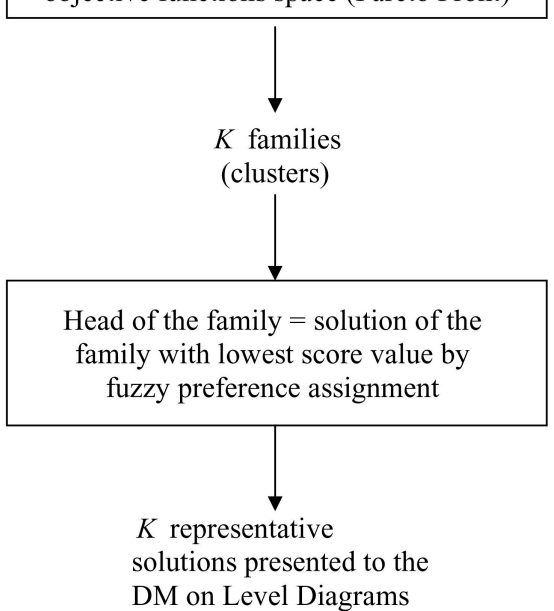

Fig. 9. Schematics of the Procedure for Reducing the Pareto Front and Set

proposed. The solutions are first clustered in "families" by subtractive clustering according to their geometric relative distance in the objective functions space (Pareto Front); the parameters driving the subtractive clustering 
are chosen to maximize the quality of the resulting Pareto Front partition. Then, a "head of the family" solution is selected as the best representative solution in each family, according to the DM preferences, by a fuzzy preference assignment procedure and a scoring system. Level Diagrams are used to represent and analyze the reduced Pareto Front thereby obtained.

The procedure has been applied to the problem of optimizing the test intervals of a nuclear power plant safety system. The results obtained show that the clustering-based reduction framework maintains the Pareto Front shape and relevant characteristics, while making it easier for the decision maker to select the final solution.

\section{REFERENCES}

[ 1 ] Blasco , X., Herrero, J.M., Sanchis, J., Martínez, M. (2008), A New Graphical Visualization of n-Dimensional Pareto Front for Decision-Making in Multiobjective Optimization, Information Science, 178: 3908-3924.

[2] Chiu, S. (1994), Fuzzy Model Identification Based on Cluster Estimation, Journal of Intelligent \& Fuzzy Systems, 2 (3)

[3] Cho, K. I., Kim, S. H. (1997) An improved Interactive hybrid method for the linear multi-objective knapsack problema, Computers Ops. Res., 24, 11: 991-1003

[4] De Boer, L., van der Wegen, L, Telgen, J. (1998), Outranking methods in support of supplier selection, European Journal of Purchasing and Supply Management, 4: 109-118.

[ 5 ] Giuggioli Busacca, P., Marseguerra, M., Zio, E. (2001), Multiobjective Optimization by Genetic Algorithms: Application to Safety Systems, Reliability Engineering and System Safety, 72: 59-74

[6] Katagiri, H., Sakawa, M, Kato, K., Nishizaki, I. (2008), Interactive multiobjective fuzzy random linear programming: Maximization of possibility and probability, European Journal of Operational Research, 188: 530-539

[ 7 ] Malakooti, B. (1988) A decision support system and a heuristic interactive approach for solving discrete multiple criteria problems, IEEE Trans. On Sys., Man and Cyber., 18: 273-284.

[ 8 ] Martorell, S., Carlos, S., Sanchez, A., Serradell, V. (2000) Constrained Optimization of Test Intervals Using a SteadyState Genetic Algorithm, Reliab, Engng Syst Safety,
67:215-32.

[9] Molina, J., Santana, L.V., Hernandez-Diaz, A.G., Coello Coello, C.A., Caballero, R. (2009), g-dominance: Reference Point Based Dominance for Multiobjective Metaheuristics, European Journal of Operational Research, 197: 658-692.

[10] NRC, US Nuclear Regulatory commission,. Rates of Initiating Events at United States Nuclear Power Plants: 1987-1995, NUREG/CR- 5750.

[11] ICRP Publication 60, (1991), 1990 recommendations of the International Commission on Radiological Protection, Annals of the ICRP, 21: 1-3.

[12] Rios Insua, D., Martin, J. (1994), Robustness Issue under Imprecise Beliefs and Preferences, Journal of Statistical Planning and Inference, 40, Issues 2-3: 383-389.

[13] Rousseeuw, P. J. (1987) Silhouettes: A graphical Aid to the Interpretation and Validation of Cluster Analysis. Journal of Computational and Applied Mathematics, 20 : 53-65.

[14] Rousseeuw P., Trauwaert E. and Kaufman L. (1989), Some Silhouette-based Graphics for Clustering Interpretation. Belgian Journal of Operations Research, Statistics and Computer Science, 29 (3).

[15] Roy, B. (1968) Classement et Choix en Presence de Points de Vue Multiples (la Methode ELECTRE), RIRO, 8: 57-75.

[16] Roy, B. (1974) Criteres Multiples et Modelisation des Preferences (l'Apport des Relations de Surclassement), Revue d'Economie Politique, 84: 1-44.

[17] Roy, B., Bouyssou, D. (1986), Comparison of two DecisionAid Models Applied to a Nuclear Power Plant Siting Example, European Journal of Operational Research, 25:200-215.

[18] Yang, J.B. (1996), Multiple Criteria Decision Making Methods and Applications, Hunan Publishing House, Changsha P.R. China.

[19] Yang, J-E., Hwang, M-J., Sung, T-Y., Jin, Y. (1999) Application of Genetic Algorithm for Reliability Allocation in Nuclear Power Plants, Reliab Engng Syst Safety, 65:22938.

[20] Yang, J.B. (2000), Minimax Reference Point Approach and its Application for Multiobjective Optimisation, European Journal of Operational Research, 126: 541-556.

[21] Zio, E., Baraldi, P., Pedroni, N. (2009), Optimal Power System Generation Scheduling by Multi-Objective Genetic Algorithms with Preferences, Reliability Engineering and System Safety, 94: 432-444.

[22] Zio, E., Bazzo, R. (2009), Multiobjective Reliability Allocation Problems by Fuzzy Preference Assignment on Level Diagrams 